\title{
Infección fúngica invasiva en pacientes inmunosuprimidos atendidos en un hospital de tercer nivel
}

\author{
Invasive fungal infection in immunosuppressed \\ patients treated in a tertiary hospital
}

Diana Rey SÁnchez, MD. ${ }^{(1)}$; Laura Bernal Vaca, MD. ${ }^{(1)}$; Melva Yomary Linares Linares, Bact. ${ }^{(2,3)}$; Claudia Marcela ParRa Giraldo, Bact. ${ }^{(2,3)}$; JaVier Ricardo Garzón ERAzo, MD.(3); SandRa Liliana ValderRama Beltrán, MD. ${ }^{(3)}$; AlejandRa CAÑAs ARboleda, MD. ${ }^{(1)}$

\section{Resumen}

ANTECEDENTES: las infecciones fúngicas invasivas son una causa importante de morbimortalidad mundial. El aumento en la incidencia de estas micosis se relaciona con el incremento de las condiciones de inmunosupresión.

OBJETIVO: Describir las características demográficas y clínicas de pacientes inmunosuprimidos tratados con antifúngicos por sospecha de infecciones fúngicas invasivas en una institución de tercer nivel.

MATERIALES Y MÉTODOS: estudio descriptivo de corte transversal, en el que se incluyeron pacientes que recibieron tratamiento antifúngico durante 2012 y 2013, por sospecha de infecciones fúngicas invasivas (aspergilosis, criptococosis, histoplasmosis y mucormicosis) y condición de inmunosupresión.

RESULTADOS: se analizaron 81 pacientes, con predominio masculino $(66,5 \%)$ y una media de edad de 44 años. El diagnóstico de base más frecuente fue neoplasia hematolinfoide seguido de infección por el virus de la inmunodeficiencia humana (VIH). La aspergilosis angioinvasiva (52\%) y la criptococosis (28\%) fueron las sospechas clínicas más frecuentes. El 43 y $85 \%$ de los pacientes tuvieron hallazgos anormales en radiografía y tomografía axial computarizada de tórax respectivamente. De aquellos con sospecha de aspergilosis, el $23 \%$ tuvo prueba de galactomanano positiva, en tanto que en aquellos con sospecha de criptococosis pulmonar, el $75 \%$ fue positivo para el antígeno capsular. El 22\% de los pacientes fallecieron y el 17\% requirieron estancia en UCI y soporte hemodinámico.

CONCLUSIÓN: es importante sospechar de forma temprana la infecciones fúngicas invasivas, así como establecer un algoritmo diagnóstico e instaurar el tratamiento antifúngico de manera anticipada para disminuir los desenlaces fatales. La dificultad en la confirmación microbiológica de las infecciones fúngicas invasivas continúa siendo una problemática local, similar a lo reportado en la literatura mundial.

Palabras clave: aspergilosis, criptococosis, histoplasmosis, inmunosuprimidos, infección fúngica invasiva.

\footnotetext{
Abstract

BACKGROUND: invasive fungal infections (IFI) are a major cause of mortality and morbidity worldwide. The increased incidence of these fungal infections are associated with an increase in immunosuppressive conditions.

OBJECTIVE: to describe demographic and clinical characteristics of immunosuppressed patients treated with antifungals for suspected invasive fungal infection (IFI) in a tertiary hospital.
}

\footnotetext{
(1)Unidad de Neumología, Departamento de Medicina Interna, Facultad de Medicina, Hospital Universitario San Ignacio, Bogotá, Colombia.

${ }^{(2)}$ Grupo de Enfermedades Infecciosas, Departamento de Microbiología, Facultad de Ciencias, Pontificia Universidad Javeriana, Bogotá, Colombia.

${ }^{(3)}$ Unidad de Infectología, Departamento de Medicina Interna, Facultad de Medicina, Hospital Universitario San Ignacio, Bogotá, Colombia.

Correspondencia: Alejandra Cañas Arboleda, correo electrónico: alejandra.canas@ javeriana.edu.co

Recibido: 10/03/16. Aceptado: 20/03/16
} 
MATERIALS AND METHODS: in this descriptive cross-sectional study, immunosuppressed patients receiving antifungal treatment during 2012 and 2013, with suspected IFI (aspergillosis, cryptococcosis, mucormicosis and histoplasmosis) were included.

RESULTS: 81 patients, predominantly men (66.5\%), with a mean age of 43.8 years were analyzed. The most common underlying conditions were lymphoproliferative disorders followed by HIV infection. Angioinvasive aspergillosis (52\%) and cryptococcosis (28\%) were most frequently suspected. $43 \%$ and $85 \%$ of patients had abnormal findings on chest radiograph and CT, respectively. $22 \%$ of the patients died and 17\% required monitoring in the ICU and hemodynamic support. Conclusion: This highlights the importance of a high index of suspicion and early diagnosis of IFI, following an algorithm that enables the timely initiation of antifungal treatment to reduce fatal outcomes. It also identifies the obstacles of getting microbiological confirmation of these pathogens; the difficulties being similar in our institution to those reported elsewhere.

Keywords: aspergillosis, cryptococcosis, histoplasmosis, immunosuppressed, invasive fungal infection.

\section{Introducción}

Las infecciones fúngicas invasivas son cada vez más frecuentes en la práctica clínica, afectan especialmente a pacientes con grados variables de inmunocompromiso y constituyen un reto para el clínico en cuanto a su abordaje diagnóstico y terapéutico. Se han catalogado como infecciones oportunistas, ya que se manifiestan en pacientes con alteraciones en la respuesta inmune $(1,2)$. La infección por VIHSIDA es considerada uno de los principales factores de riesgo para adquirir infecciones fúngicas invasivas, sin dejar a un lado los pacientes con neoplasia hematológica expuestos a esquemas de quimioterapia. Otros grupos de riesgo son aquellos pacientes con uso de terapia biológica y trasplantados (3). Pese a que tienen una incidencia comparativamente menor a la de las infecciones causadas por bacterias multirresistentes y virus, el impacto negativo para el paciente y los costos elevados de su tratamiento, hacen de estas un verdadero reto para la comunidad médica (4).

Aun cuando se dispone de nuevos fármacos antimicóticos con mejores características farmacológicas, el pronóstico de los pacientes con infecciones fúngicas invasivas continúa siendo desalentador, debido al diagnóstico tardío y a la demora en el inicio del tratamiento antifúngico (5). Estas entidades tienen la capacidad de afectar diferentes órganos como pulmón, sistema nervioso central, vías respiratorias altas, piel, hígado y bazo, lo cual condiciona un compromiso grave en el paciente, que requiere por lo general manejo en unidades de cuidado intensivo y genera altos costos para el sistema de salud.
La identificación histopatológica de los agentes etiológicos en muestras de tejidos y cultivos son los métodos más utilizados para el diagnóstico (6). Infortunadamente, las poblaciones de pacientes con mayor riesgo de infecciones fúngicas invasivas son también aquellas con alto riesgo de complicaciones asociadas a biopsias invasivas, lo que limita la utilidad histopatológica $(7,8)$. La biopsia no suele ser una opción para los pacientes con neutropenia y sospecha de infecciones fúngicas invasivas como aspergilosis, debido a que estos también son propensos a cursar con trombocitopenia con alto riesgo de sangrado (8).

Es así como la European Organization for Research and Treatment of Cancer (EORTC) y el National Institute of Allergy and Infectious Diseases (NIAID) han considerado tres pilares para el diagnóstico de las infecciones fúngicas invasivas en pacientes con cáncer y trasplante de precursores hematopoyéticos, los cuales permiten clasificar estas infecciones como confirmadas, probables o posibles (1):
a. Factores predisponentes en el hospedero.
b. Elementos clínicos-radiológicos.
c. Hallazgos de laboratorio.

De esta forma, teniendo en cuenta el perfil de complejidad de los pacientes atendidos en instituciones de tercer nivel y el gran número de estos con riesgo de inmunosupresión, es deber velar por fortalecer la prestación de servicios integrales en la clínica y hacer un diagnóstico oportuno. Se planteó, 
en consecuencia, describir características demográficas, clínicas, abordaje diagnóstico y desenlaces de pacientes inmunosuprimidos con sospecha de infecciones fúngicas invasivas en una institución de tercer nivel en Bogotá, Colombia, durante un periodo de dos años.

\section{Materiales y métodos}

Estudio descriptivo de corte transversal, llevado a cabo desde enero de 2012 a diciembre de 2013, en un hospital de tercer nivel de atención en Bogotá, Colombia, en el que incluyeron pacientes inmunosuprimidos mayores de 18 años hospitalizados, con manejo antifúngico y que cursaran con sospecha de infecciones fúngicas invasivas por aspergilosis angioinvasiva, criptococosis, histoplasmosis o mucormicosis.

Se definió inmunosupresión por: infección por el virus de la inmunodeficiencia humana (VIH) con recuento de CD4 menor de $500 \mathrm{cel} / \mathrm{u}$-, neutropenia menor 500 neutrófilos $/ \mathrm{mm}^{3}$, trasplante alogénico de progenitores hematopoyéticos, trasplante de órgano sólido, dosis de corticoides mayor $0,5 \mathrm{mg} / \mathrm{kg} / \mathrm{día}$ (equivalentes de prednisona) durante más de tres semanas, tratamiento con quimioterapia de inducción o de rescate en leucemias y linfomas, tratamiento con anti-linfocitos $\mathrm{T}$ como ciclosporina, anti-TNF, o análogos nucleósidos durante los tres meses previos e inmunodeficiencia grave congénita (enfermedad granulomatosa crónica). El criterio de infección fúngica invasiva se definió con base en los criterios EORTC/MSG como infecciones fúngicas invasivas "confirmada, "probable" o "posible" $(3,9)$.

Se excluyeron pacientes que recibieron antifúngicos por otra indicación diferente al manejo de infecciones fúngicas invasivas o que no cumplieran con alguno de los estados de inmunosupresión mencionados. No se hizo ningún tipo de intervención en los pacientes ni se solicitaron paraclínicos adicionales. Se excluyeron pacientes con infección por Pneumocystis jirovecii.

Se midieron las variables durante el evento de infecciones fúngicas invasivas, incluyendo variables demográficas como: edad, sexo, talla, peso, sitio de procedencia e índice de masa corporal (IMC). Se tuvo en cuenta el diagnóstico de los pacientes que condicionara su estado de inmunosupresión y el tipo de infecciones fúngicas invasivas que se sospechó. Se registraron los síntomas generales y respiratorios con los que cursaron durante el evento, así como los hallazgos paraclínicos: recuento absoluto de neutrófilos, hallazgos radiológicos, resultado de estudios microbiológicos e histopatológicos, resultados de test indirectos de detección de antígeno galactomanano, así como el tratamiento antifúngico recibido. El desenlace medido fue la respuesta clínica, establecida como muerte durante la hospitalización y/o necesidad de ingreso a la Unidad de Cuidados Intensivos (UCI).

\section{Análisis estadístico}

Las variables demográficas, así como la frecuencia de cada uno de los factores de riesgo, se describieron utilizando medidas de tendencia central y de dispersión cuando era el caso (media y desviación estándar para variables continuas), o tablas de frecuencia. Se evaluó la asociación entre cada uno de los factores de riesgo y la presencia de infecciones fúngicas invasivas a través de los criterios de la EORTC, así como la asociación entre los factores de riesgo y mortalidad y/o admisión a UCI. Se usó el programa estadístico STATA.

\section{Resultados}

\section{Descripción general}

Se encontraron 550 pacientes que recibieron tratamiento antifúngico, incluyendo fluconazol, anfotericina $\mathrm{B}$, itraconazol, posaconazol, caspofungina y voriconazol, de los cuales se incluyeron 80 que cumplían con los criterios de inmunosupresión y cursaban con sospecha de infecciones fúngicas invasivas. Una paciente cursó con dos eventos de infecciones fúngicas invasivas, con lo cual se incluyeron para el análisis 81 eventos. Se excluyeron 470 pacientes que no cumplían con los criterios de inclusión o recibían tratamiento antifúngico por otra indicación. En la tabla 1 se describen las características demográficas y clínicas. 
Tabla 1. Caracterización de pacientes con sospecha clínica de infección fúngica invasiva.

\begin{tabular}{|c|c|}
\hline Característica & $\begin{array}{c}\text { Número de } \\
\text { pacientes (\%) }\end{array}$ \\
\hline \multicolumn{2}{|l|}{ Género } \\
\hline Masculino & $53(66,2)$ \\
\hline Femenino & $27(33,7)$ \\
\hline Edad promedio & 44 años \\
\hline \multicolumn{2}{|l|}{ Zona urbana } \\
\hline IMC promedio & $74(92,0) 22$ \\
\hline Tabaquismo & $11(13,6)$ \\
\hline \multicolumn{2}{|l|}{ Tratamiento inmunosupresor } \\
\hline Quimioterapia & $18(22,2)$ \\
\hline Esteroide + otro inmunosupresor & $21(26,0)$ \\
\hline Terapia biológica & $2(2,5)$ \\
\hline \multicolumn{2}{|l|}{ Diagnóstico de base } \\
\hline Enfermedad hematológica & $44(54,3)$ \\
\hline LLA & $13(16,0)$ \\
\hline LMA & $12(14,8)$ \\
\hline Linfoma no Hodking & $6(7,4)$ \\
\hline Síndrome mielodisplásico & $5(6,2)$ \\
\hline Linfoma Hodking & $5(6,2)$ \\
\hline Mieloma múltiple & $3(3,7)$ \\
\hline Infección por VIH & $32(39,5)$ \\
\hline Enfermedad autoinmune & $3(3,7)$ \\
\hline Trasplante cardiaco & $1(1,2)$ \\
\hline Tumor sólido & $1(1,2)$ \\
\hline \multicolumn{2}{|l|}{ Recuento de CD4 promedio } \\
\hline$\left(\mathrm{cel} / \mathrm{mm}^{3}\right)$ & 48,7 \\
\hline
\end{tabular}

Del grupo de pacientes analizados, el 54\% $(n=44)$ tenía como diagnóstico de base neoplasia hematolinfoide, seguido de infección por VIH en un $39 \%$ $(n=32)$, de los cuales el 56\% $(n=18)$ recibía tratamiento antirretroviral. La manifestación clínica más frecuente fue fiebre en el $55 \%$ de los pacientes, fiebre y cefalea en el $12 \%$ y fiebre, vómito y cefalea en el $8 \%$. Es importante resaltar que el $18 \%$ de los pacientes no presentaba síntomas generales.

\section{Descripción de la infección fúngica invasiva}

\section{Aspergilosis angioinvasiva}

Fue la condición que se sospechó con mayor frecuencia en el grupo de pacientes analizados (52\%) $(n=42)$. Todos cursaban con neoplasia hematolinfoide, excepto un caso con diagnóstico de infección por VIH.

Al aplicar los criterios EORTC, se logró establecer infección fúngica invasiva "confirmada" en el 9\% $(n=4)$ de los pacientes, "probable" en el 20\% $(n=9)$ y "posible" en el 70\% (n=28). El 31,7\% tenía como diagnóstico leucemia linfoide aguda, 26,8\% leucemia mieloide aguda, 14,6\% linfoma no Hodgkin, 9,7\% linfoma Hodgkin, 9,7\% síndrome mielodisplásico y $7,3 \%$ mieloma múltiple.

En cuanto a las manifestaciones clínicas, el $84 \%$ refirió fiebre, el $73 \%$ tos, el $50 \%$ disnea y el $7 \%$ sinusitis concomitante. Se realizó radiografía de tórax a todos los pacientes, y se encontraron hallazgos anormales solo en la mitad (infiltrados alveolares en el $61 \%$, infiltrados intersticiales en el $23 \%$, derrame pleural en el 15\% y nódulos en el 7,6\%).

A todos se les hizo tomografía axial computarizada de tórax de alta resolución (TACAR), en la que se evidenciaron anormalidades en todos los estudios, $84 \%$ tenían nódulos con halo en vidrio esmerilado, 34\% infiltrados alveolares, $23 \%$ infiltrados en vidrio esmerilado, $15 \%$ derrame pleural, $7,6 \%$ infiltrados intersticiales y 7,6\% infiltrados tipo “árbol en gemación”.

Se realizó fibrobroncoscopia a la mitad de los pacientes y se hallaron anormalidades en el $38 \%$ (signos de traqueobronquitis y hemorragia alveolar en 3 y 2 pacientes respectivamente). Se hizo biopsia pulmonar a 3 pacientes, sin encontrar hallazgos histopatológicos sugestivos de infecciones fúngicas invasivas. Una de las pacientes con leucemia linfoide aguda, presentó aspergilosis en el tracto digestivo, confirmada por biopsia. 
Solo se efectuó estudio con galactomanano en 39 pacientes, y se obtuvieron resultados positivos en el $23 \%$ de los casos $(n=9)$.

En cuanto al manejo antifúngico, la mayoría recibió voriconazol, seguido por anfotericina (11\%), caspofungina (3\%) y posaconazol (3\%). 4 pacientes se manejaron con terapia combinada, 2 recibieron voriconazol y caspofungina y 2 voriconazol y anfotericina B. De este grupo de pacientes fallecieron 8 .

\section{Criptococosis}

Fue la segunda micosis sospechada en un $28,3 \%$ $(n=23)$. De este grupo el $34 \%(n=8)$ de los pacientes cursaron con criptococosis pulmonar, todos de género masculino. Siete tenían infección por VIH como patología de base y uno sarcoidosis. Los pacientes VIH tenían un conteo de CD4 promedio de 69, y sólo uno se encontraba en terapia antirretroviral y profilaxis con fluconazol. Todos tenían carga viral detectable y en 3 casos esta fue la enfermedad definitoria de SIDA. 6 presentaron compromiso meníngeo y consultaron por cefalea y fiebre, 2 referían tos $\mathrm{y}$ disnea a la revisión por sistemas. A todos se les realizó radiografía de tórax, que fue normal en todos los casos, excepto en 2 que presentaron infiltrados reticulares bilaterales.

En los 8 pacientes se evidenciaron nódulos en la tomografía de tórax: cuatro nódulos cavilados, dos nódulos con halo en vidrio esmerilado, dos tenían además consolidación, y en tres se observaron adenomegalias mediastinales.

Cinco de los 8 pacientes tenían hemocultivos positivos para Cryptococcus, y seis tuvieron látex positivo en líquido cefalorraquídeo (LCR), con cinco cultivos positivos en líquido cefalorraquídeo. Sólo a 3 pacientes se les realizó fibrobroncoscopia, todas con tinciones directas negativas, dos con biopsias transbronquiales en las cuales se evidenció compromiso por Cryptococcus, y adicionalmente una de ellas con visualización de estructuras micóticas en el citológico. En el resto de pacientes,

habiéndose aislado Cryptococcus en sangre o del sistema nervioso central, el compromiso pulmonar se atribuyó a criptococosis diseminada, no considerándose necesaria la realización de estudios adicionales a nivel pulmonar. Todos los pacientes con VIH recibieron anfotericina B y 2 recibieron además 5-fluocitosina. Todos completaron el tratamiento y tuvieron mejoría progresiva del cuadro hasta el egreso.

El caso de sarcoidosis como diagnóstico de base, dificultó la pronta identificación de la etiología del cuadro, no recibió tratamiento antifúngico y falleció. Cabe mencionar que para este paciente se obtuvo el aislamiento de Cryptococcus postmortem.

\section{Histoplasmosis}

Esta micosis se sospechó en el 11\% $(n=9)$ de los pacientes analizados. Todos tenían infección por VIH, excepto uno con diagnóstico de síndrome mielodisplásico. 4 pacientes fueron confirmados por histopatología, y en 1 de estos casos se recuperó Histoplasma capsulatum. Las tomografías mostraron en 8 pacientes infiltrados en "árbol en gemación, lo cual orientó el diagnóstico. En el paciente con síndrome mielodisplásico se encontró coinfección con Cryptococcus. Todos fueron tratados con anfotericina B, de los cuales fallecieron 3, entre ellos el paciente con síndrome mielodisplásico.

\section{Mucormicosis}

En el 2,4\% (n=2) de los casos se sospechó esta micosis, ambos cursaban con síntomas de sinusitis y disnea. En la TACAR se evidenciaron nódulos de densidad de tejidos blandos con halo en vidrio esmerilado. Sin embargo, un solo paciente fue llevado a fibrobroncospia y lavado broncoalveolar, con citología positiva para estructuras hifales pero con $\mathrm{KOH}$ y cultivo negativo. Este paciente recibió tratamiento con posaconazol y el que no fue llevado a broncoscopia recibió tratamiento inicial con anfotericina $\mathrm{B}$ y posteriormente con voriconazol. Ambos evolucionaron de manera satisfactoria.

\section{Discusión}

Las infecciones fúngicas invasivas son una causa importante de morbilidad y mortalidad mundial. El aumento de la incidencia y la severidad de estas micosis pueden ser directamente atribuibles a un 
incremento de las poblaciones en riesgo, que incluye pacientes con cáncer, SIDA, receptores de órganos y en general, inmunosuprimidos. Este estudio descriptivo permitió caracterizar la población que presenta esta complicación en una institución de tercer nivel, así como las herramientas diagnósticas más utilizadas y su comportamiento en este grupo de pacientes.

Se determinó que el mayor número de pacientes cursaba con neoplasia hematolinfoide, seguido por infección por VIH. En este último grupo solo la mitad recibía tratamiento antirretroviral, lo que está a favor de los bajos conteos de CD4 que se documentaron en estos pacientes, cuyo promedio fue de $48 \mathrm{cel} / \mathrm{mm}^{3}$, dato que coincide con lo reportado por la literatura sobre el mayor riesgo de infecciones fúngicas invasivas con recuentos de CD4 menores a $50 \mathrm{cel} / \mathrm{mm}^{3}$.

Se encontraron diferentes tipos de desórdenes hematolinfoides, incluyendo leucemias agudas, linfomas y síndrome mielodisplásico, además de aspergilosis angioinvasiva como la infección fúngica invasiva más sospechada. La mayoría presentó sintomatología inespecífica con mal rendimiento de la radiografía de tórax. En cuanto a la tomografía de tórax, en la mayoría de casos, los hallazgos fueron los habitualmente descritos para aspergilosis invasiva, con presencia de nódulos con halo en vidrio esmerilado.

Las pruebas convencionales de laboratorio utilizadas para la confirmación de las infecciones fúngicas invasivas en la mayoría de casos resultan inespecíficas por la dificultad frente a la interpretación de los resultados; por ejemplo, el examen directo con $\mathrm{KOH}$, no tiene buen rendimiento diagnóstico. La toma de biopsias, que puede tener mejor rendimiento, es de difícil realización, especialmente por las condiciones en las que con frecuencia se encuentran estos pacientes que contraindican el procedimiento. Esto ha llevado a utilizar otras metodologías diagnósticas como la detección de antígeno capsular para Cryptococcus y detección de galactomanano en pacientes neutropénicos con aspergilosis. En este estudio se obtuvo una positividad del $75 \%$ en las pruebas de antígeno capsular para Cryptococcus y un del 23\% para la prueba de galactomanano; este dato posiblemente se deba a que no a todos los pacientes se les realizó el examen seriado.

Sin lugar a dudas, el diagnóstico micológico se fundamenta en el cultivo para el aislamiento e identificación del agente infeccioso y la realización de pruebas de susceptibilidad; sin embargo, en este estudio el $68 \%$ de los cultivos realizados fueron negativos. La mayoría de muestras procesadas correspondían a hemocultivos, los cuales no han mostrado eficiencia para la recuperación de la mayoría de hongos filamentosos. Estos resultados concuerdan con lo reportado en la literatura, en la que los hemocultivos negativos son la regla general para las infecciones fúngicas invasivas, y por lo tanto tienen baja utilidad para su diagnóstico $(10,11)$.

La mortalidad fue más baja que lo reportado en la literatura, en cuyo caso se alcanzan tasas de mortalidad hasta del $80 \%$ (12). Este resultado puede estar asociado con el inicio rápido del manejo antifúngico frente a la sospecha de infecciones fúngicas invasivas.

En este estudio se resalta la importancia de sospechar en forma temprana esta entidad, para así disminuir su morbimortalidad, siguiendo un algoritmo diagnóstico preciso para documentar la infecciones fúngicas invasivas. Como debilidad del estudio, se anota que sólo se incluyeron pacientes de forma retrospectiva y aquellos que recibían terapia antifúngica, lo cual excluye pacientes que cursaron con infecciones fúngicas invasivas y no recibieron tratamiento antifúngico oportuno.

Estos resultados permiten evidenciar la necesidad de continuar líneas de investigación que fortalezcan las bases ya existentes en el área de microbiología en cuanto a identificación y pruebas de susceptibilidad para hongos.

Se plantea en particular que es fundamental realizar un diagnóstico precoz de la aspergilosis invasiva para el inicio oportuno de terapia antifúngica y así mejorar las condiciones en pacientes de alto riesgo. El uso de los biomarcadores sensibles, incluyendo los ensayos no invasivos para galactomanano y 1-3D - glucano, reduce el uso de agentes 
antifúngicos innecesarios. Por lo anterior, se plantea la necesidad de evaluar el momento de la positividad y la sensibilidad de un dispositivo de flujo lateral en pacientes inmunosuprimidos con sospecha de aspergilosis pulmonar invasiva para comparar directamente estos resultados con los obtenidos mediante el galactomanano y 1-3D- glucano, estudio que se lleva a cabo en nuestra institución.

\section{Agradecimientos}

A la Dra. Laura Kattah, residente de Medicina Interna de la Pontificia Universidad Javeriana.

\section{Conflictos de interés}

Los autores declaran que están de acuerdo con los resultados publicados en este artículo y que no existe conflicto de intereses.

\section{Bibliografía}

1. Hoenigl M, Strenger V, Buzina W, Valentin T, Koidl C, Wölfler A, et al. European Organization for the Research and Treatment of Cancer/Mycoses Study Group (EORTC/ MSG) host factors and invasive fungal infections in patients with haematological malignancies. J Antimicrob Chemother. 2012;67(8):2029-33.

2. Perusquía-Ortiz AM, Vázquez-González D, Bonifaz A. Opportunistic filamentous mycoses: aspergillosis, mucormycosis, phaeohyphomycosis and hyalohyphomycosis. J Dtsch Dermatol Ges. 2012;10(9):611-21.

3. Warnock DW. Trends in the epidemiology of invasive fungal infections. Japanese. J Med Mycol. 2007;48(1):1-12.
4. Moreno A, Cervera C, Fortú J, Blanes M, Montejo E, Abradelo M, et al. The OLT-HIV-FIPSE Cohort Investigators. Epidemiology and Outcome of Infections in Human Immunodeficiency Virus/Hepatitis C Virus-Coinfected Liver Transplant Recipients: A FIPSE/GESIDA Prospective Cohort Study. Liver Transplant. 2012;18:70-82.

5. Springer J, Einsele H, Loeffler J. Molecular techniques in the diagnosis of deep and systemic mycosis. Clin Dermatol. 2012;30(6): 651-6.

6. Wengenack NL, Binnicker MJ. Fungal molecular diagnostics. Clin Chest Med. 2009;30(2):391-408.

7. Alexander BD. Diagnosis of fungal infection: new technologies for the mycology laboratory. Transpl Infect Dis. 2002;4Supl.3:32-7.

8. Vehreschild, JJ Ruping, MJ Steinbach, A Cornely O. Diagnosis and treatment of fungal infections in allogeneic stem cell and solid organ transplant recipients. Expert Opin Pharmacother. 2010;11(1):95-113.

9. De Pauw B, Wals T, Donnelly J, Stevens D, Edwards J, Calandra $\mathrm{T}$, et al. Revised definitions of invasive fungal disease from the European Organization for Research and Treatment of Cancer/Invasive Fungal Infections Cooperative Group and the National Institute of Allergy and Infectious Diseases Mycoses Study Group (EORTC/MSG). Clin Infect Dis. 2008;46(12):1813-21.

10. Barnes PD, Marr K A. Risks, diagnosis and outcomes of invasive fungal infections in haematopoietic stem cell transplant recipients. Br J Haematol. 2007;139:519-31.

11. Sales PU. Aspergillosis: From diagnosis to treatment. EN: J Bras Pneumol. 2009;35:1238-44.

12. Mori YA, Kanda Y, Oshima K, Kako S, Shinohara A, Nakasone $\mathrm{H}$, et al. False- positive Aspergillus galactomannan antigenaemia after haematopoietic stem cell transplantation. J Antimicrob Chemother. 2008;61(2):411-6. 